\title{
Las nuevas actitudes de los docentes tras la emergencia sanitaria
}

Los estudios sobre actitudes desarrollados desde finales del siglo pasado han determinado que se fundamentan por tres componentes: el cognitivo (relacionados con objetos de estudio y las construcciones teóricas); el afectivo (relacionado con los sentimientos sobre el objeto) y el comportamental (relacionado con las conductas y acciones sobre el objeto de estudio).

El mundo tuvo que realizar grandes esfuerzos para adaptarse a las necesidades derivadas de la COVID-19, que forzó a la educación y a los docentes a la configuración de nuevos escenarios, materiales y constructos. Adaptarse supuso repensar cuales son las prioridades de una educación para el siglo XXI, pero, sobre todo supuso desarrollar actitudes propias derivadas de la emergencia sanitaria.

Desde esta perspectiva, las instituciones, las políticas y en general las comunidades académicas han desarrollado nuevas formas de relacionarse con los objetos de estudio, nuevas formas de introducir los conceptos, de evaluar los aprendizajes y en especial nuevas maneras de comportarse frente a los procesos de enseñanza-aprendizaje, lo que se traduce en nuevas actitudes desarrolladas desde y para una educación en confinamiento.

Hace muy poco tiempo en las políticas publicas y en las proyecciones se hablaba de formar a las nuevas generaciones, sin pensar que la COVID-19 nos mostró que no es posible determinar o predecir como serán las nuevas generaciones, cuáles serán los principales problemas o cuáles serán las necesidades sociales a las que la ciencia y la tecnología deberá atender, necesitamos repensar la forma en la cual validamos los conocimientos, cómo acercamos a los estudiantes a la autorregulación y al desarrollo de habilidades propias de las didácticas específicas. Atrás se quedaron las antiguas formas de evaluar y la contextualización de los contenidos. En esta nueva era la globalización es una realidad, las necesidades y los enfoques ya no pueden ser locales o particulares, necesitamos construir a partir de esta experiencia forzada diferentes maneras de relacionarnos con los objetos de estudio y nuevas e innovadoras formas de construir conocimiento a partir de modelos y realidades.

La Universidad Distrital Francisco José de Caldas así lo ha entendido y tras la declaratoria de emergencia sanitaria realizó procesos de formación y adaptación a toda su comunidad académica y sin perder el enfoque misional que ha permitido que la continuidad de la docencia, la investigación y la extensión.

Mg. William Fernando Castrillón Cardona

Dra. Adriana Patricia Gallego Torres

Vicerrector Académico

Editora Revista Científica 